\title{
Erratum to: A database of body-only computer-generated pictures of women for body-image studies: Development and preliminary validation
}

\author{
Joanna M. Moussally ${ }^{1,2} \cdot$ Lucien Rochat $^{1,2} \cdot$ Andrés Posada $^{3}$. \\ Martial Van der Linden ${ }^{1,2,4}$
}

Published online: 18 April 2017

(C) Psychonomic Society, Inc. 2017

Erratum to: Behav Res

DOI 10.3758/s13428-016-0703-7

Published 49_1 pp. 172-183

There is an error in the second sentence of Footnote 3 on page 175 .

The correct sentence should read:

A pilot study with 40 participants (24 women, 16 men) aged 19 to 68 years old showed that the eight adjectives. . .

The online version of the original article can be found at http://dx.doi.org/ 10.3758/s13428-016-0703-7

Joanna M. Moussally

Joanna.Moussally@unige.ch

1 Cognitive Psychopathology and Neuropsychology Unit, Psychology Department University of Geneva, Geneva, Switzerland

2 Swiss Center for Affective Sciences, University of Geneva, Geneva, Switzerland

3 Psychology Department, University of Geneva, Geneva, Switzerland

4 Cognitive Psychopathology Unit, Cognitive Sciences Department, University of Liège, Liège, Belgium 